\title{
Globe
}

Revue internationale d'études québécoises

\section{Lise Bizzoni et Cécile Prévost-Thomas (dir.), avec la collaboration de Dany Saint-Laurent : La chanson francophone engagée, Montréal, Triptyque, 2008}

\section{Andrea Oberhuber}

Volume 12, numéro 2, 2009

URI : https://id.erudit.org/iderudit/1000720ar

DOI : https://doi.org/10.7202/1000720ar

Aller au sommaire du numéro

Éditeur(s)

Globe, Revue internationale d'études québécoises

ISSN

1481-5869 (imprimé)

1923-8231 (numérique)

Découvrir la revue

Citer ce compte rendu

Oberhuber, A. (2009). Compte rendu de [Lise Bizzoni et Cécile Prévost-Thomas (dir.), avec la collaboration de Dany Saint-Laurent : La chanson francophone engagée, Montréal, Triptyque, 2008]. Globe, 12(2), 225-230.

https://doi.org/10.7202/1000720ar d'utilisation que vous pouvez consulter en ligne.

https://apropos.erudit.org/fr/usagers/politique-dutilisation/ 
satisfasse tous les membres. La part réservée à la politique reste extrêmement importante, tant dans la Déclaration que dans les Résolutions. Il est à noter par ailleurs une insistance très nette sur la défense et la promotion du français; le secrétaire général est par exemple invité à " entamer les démarches visant à conclure un pacte linguistique avec les États ou gouvernements qui le souhaitent" (Résolution sur la langue française, adoptée lors du Sommet de Québec, http://www.francophonie.org/IMG/pdf/resolution-quebec-20083.pdf [26 janvier 2010]), ce qui peut apparaître comme une première étape, non suffisante certes mais nécessaire, vers un renforcement de l'identité culturelle de la Francophonie. Entièrement oubliée des rédacteurs de ce collectif, la question de l'environnement est très présente dans la Déclaration. En revanche, la dimension économique reste clairement le parent pauvre des projets de la Francophonie, surtout étant donné les enjeux soulevés dans ce livre: il est par exemple indiqué que l'assemblée prendra, "lors du $\mathrm{XIII}^{\mathrm{e}}$ Sommet, une décision quant à l'opportunité de tenir une deuxième Conférence des ministres de l'Économie et des Finances ou d'autres décideurs économiques de l'espace francophone" (Déclaration de Québec, paragraphe 37, http://www.francophonie.org/IMG/pdf/decl-quebec-20083.pdf [26 janvier 2010])...

Tristan Leperlier École Normale Supérieure

Paris IV Sorbonne Sciences Po Paris

\section{Lise Bizzoni et Cécile Prévost-Thomas (dir.), avec la collaboration de Dany Saint-Laurent La chanson francophone engagée Montréal, Triptyque, 2008.}

Les premières années du nouveau millénaire auront été plutôt fécondes en monographies consacrées à des chanteurs ou chanteuses, de même qu'en publications savantes sur la chanson d'expression française. Que l'on songe à Plume Latraverse, masqué / démasqué de Mario Leduc (2003), à La chanson réaliste de Catherine Dutheil (2004), aux Mots de Barbara (2004) et à l'Esthétique de la chanson française contemporaine de Joël July (2007) ou à 
Chanson. L'art de fixer l'air du temps de Stéphane Hirschi (2008) ${ }^{11}$, tous ces ouvrages contribuent à faire valoir le " $9^{\mathrm{c}}$ art", pour utiliser ce terme créé par Angèle Guller, en tant que genre à part entière, comme objet d'étude légitimé. Les approches du genre de la "chanson" tiennent aujourd'hui mieux compte des spécificités génériques que dans le passé, qui voyait s'appliquer à la chanson des méthodes littéraires, comme celles pratiquées sur des textes poétiques, ou des analyses musicologiques, mais rarement des lectures croisées.

Le collectif La chanson francophone engagée, sous la direction de Lise Bizzoni et de Cécile Prévost-Thomas, s'inscrit explicitement dans cette nouvelle approche multidisciplinaire puisque l'ouvrage réunit sept contributions signées par des musicologues, des littéraires, une historienne et une sociologue. Issue d'un colloque étudiant organisé en 2005 à l'Université du Québec à Montréal sous la thématique double "La chanson francophone contemporaine et engagée ", la présente étude affiche d'emblée, c'est-à-dire dès le nouveau titre, sa volonté de resserrer les liens autour d'une notion clé, soit celle de l'engagement - social, politique ou esthétique - d'une cuvre ou de son créateur. Cette chanson "engagée", insistent les responsables du collectif dans l'introduction, est "[p]résente tout au long de l'Histoire de l'humanité" (p. 8), tout comme elle occupe une place bien plus importante dans la production chansonnière contemporaine que le discours social et médiatique, qui lui accorde bien peu d'intérêt, pourrait nous le faire croire (p. 10). C'est donc justement pour faire écho à une présence réelle, tout au long du $\mathrm{XX}^{\mathrm{e}}$ siècle certes mais également aujourd'hui, de diverses facettes de ce type de chanson de contestation dans le champ culturel, pour remédier au manque de visibilité dont semble souffrir cette chanson, que les auteurs de $L a$ chanson francophone engagée se proposent d'examiner "les objectifs, les modalités et les formes " d'un "nouvel engagement", déterminé par une "réalité sociale, intellectuelle et artistique» révolue (p. 15). Peut-on s'intéresser à la chanson engagée, à celle qui, depuis les mazarinades, accuse, revendique, incite à l'action, souhaite subvertir le système en place, sans se positionner soi-même dans le débat, qui revient tel un leitmotiv, sur l'engagement de l'artiste et de l'intellectuel dans la cité ? Lise Bizzoni et Cécile

11. Dans l'ordre: Mario LEDUC, Plume Latraverse, masquéldémasqué, Montréal, Triptyque, 2003; Catherine DUTHEIL, La chanson realiste. Sociologie d'un genre, Paris, L'Harmattan, 2004 ; Joël JULY, Les mots de Barbara, Aix-en-Provence-Marseille, Publications de l'Université de Provence, 2004 et Esthérique de la chanson française contemporaine, Paris, L'Harmatian, 2007; Stéphane HIRSCHI, Chanson. L'art de fuxer l'air du temps: de Beranger à Mano Solo, Valenciennes, Presses universitaires de Valenciennes, 2008. 
Prévost-Thomas avouent expressis verbis à la fin de l'introduction vouloir prolonger le "débat diffusé par la presse québécoise au printemps 2004 sur la question de la chanson engagée d'aujourd'hui " (p. 18-19), en démontrant "l'existence plurielle d'une chanson engagée", en illustrant à travers les diverses analyses rassemblées dans le collectif que la chanson peut être "un art engagé", que "les artistes peuvent remplir une fonction d'utilité sociale" (p. 19). C'est ce que l'on pourrait appeler une véritable profession de foi de la part de deux jeunes chercheures qui se donnent pour but d'élargir la notion d'engagement et d'ainsi contribuer à une connaissance plurielle de l'objet «chanson". Le ton est donné.

Le collectif rassemble en tout, répétons-le, sept articles qui se suivent sans être regroupés en parties; le lecteur attentif comprend cependant vite qu'une perspective historique, voire chronologique, a présidé au regroupement des diverses approches et réflexions sur la chanson engagée. Ainsi, les trois premiers articles explorent les liens entre "engagement" et "chanson" d'un point de vue musicologique. Sandria $P$. Bouliane s'intéresse à l'émergence d'une industrie phonographique au Canada français dans la première moitié du XXe siècle lui permettant, dans la foulée des travaux de Robert Thérien, de retracer l'histoire de l'enregistrement d'une chanson populaire d'abord canadienne-française puis québécoise liée intimement, par le choix de la langue et de certains sujets, à un discours identitaire. Dans une excellente étude consacrée au folklore comme "arme de persuasion dans la musique populaire québécoise avant et après l'âge d'or des chansonniers" tel est le titre de l'article -, Luc Bellemare explore, à partir de l'exemple de "La bonne chanson" de l'abbé Gadbois, la relation qui existe selon lui entre le folklore et le nationalisme, aboutissant à ce qu'il appelle un "folklore nationalisant" (p. 40); dans un second temps, il démontre, à travers une analyse finement menée, que certains groupes québécois contemporains tels Loco Locass, Mes aïeux et Les Cowboys fringants, dont il retient la chanson "En attendant (le reel de nos gens)", renouent avec le folklore d'autrefois afin de doter leurs chansons de "couleurs folkloriques dans le texte et l'interprétation musicale" (p. 55). Audrée Descheneaux propose, pour terminer la ronde des approches musicologiques, une étude comparée de deux chansons: avec l'analyse de "Capital" de Vulgaires Machins et de "Pauvres riches" de Tomàs Jensen $\&$ Les faux-monnayeurs, on reste dans la production québécoise contemporaine. L'auteure montre, schémas musicaux à l'appui, que la syntaxe musicale permet dans les deux cas de véhiculer le message social et politique: «la structure musicale, note-t-elle, agit comme support du discours engagé en le propulsant vers un phénomène d'adhésion 
spontanée du public" (p. 83). Si l'analyse des deux exemples peut paraître convaincante, les conclusions générales que tire Descheneaux à la toute fin de son article paraissent trop hâtives; elles exigeraient un corpus plus élargi, davantage diversifié.

Avec l'article de Dany Saint-Laurent, la réflexion sur la "chanson engagée " change de direction. Se réclamant d'une formation en création littéraire, puis en études littéraires, l'auteure insiste sur la "trinité générique" (p. 89) que constitue la "rapoésie» (rap, poésie, manifeste) de Loco Locass. Au lieu d'explorer cette nouvelle appellation générique afin de mieux l'illustrer dans une visée poétique, Dany Saint-Laurent se penche sur les images des pochettes de disques du groupe québécois et leur impact sur l'auditeur. Les digressions sont nombreuses, la conclusion d'une "stratégie cohérente" (p. 104) d'un album à l'autre paraît sans surprise. On aurait pu s'attendre à ce que la seconde analyse littéraire, celle de Lise Bizzoni, suive l'article de Saint-Laurent. Les deux études auraient pu former une certaine unité, mais l'écart aurait peut-être été trop grand. Qu'il nous soit permis, exceptionnellement, de briser l'ordre établi par les responsables du collectif pour placer dans le contexte des approches littéraires l'analyse très informée et fort originale que propose Bizzoni : $s$ 'inspirant des travaux de Dominique Garand, elle place l'engagement social et politique de plusieurs groupes français (Zebda, Massilia Sound Système, Oneyed Jack, Ceux d'en face, le collectif Motivés) dans le cadre théorique des stratégies thétoriques au sens propre, en recourant notamment aux notions d'ethos, de pathos et de posture. L'exemple de la reprise et de la réappropriation du célèbre hymne de la Résistance, "Le chant des partisans», lui permet de mettre en évidence une double stratégie: (r)éveiller la conscience du public et responsabiliser celui-ci tout en le divertissant. Il est vrai que, comme le souligne Bizzoni, contrairement à l'année 1943, dans les années 1990, "la cause ne suffit plus pour mobiliser les troupes" (p. 168). Dans la reprise du "Chant" par le Taktikollectif, par le biais de nouveaux arrangements musicaux, un rythme et un tempo accélérés et des performances festives lors des grandes manifestations publiques, "Le chant des partisans" se voit complètement transformé. Aussi paradoxal que cela puisse paraître, face à de nouveaux dangers, soixante ans plus tard, l'engagement politique peut s'exprimer dans l'allégresse.

Regroupons, pour terminer, parce que les deux études s'inscrivent dans le champ des sciences sociales, l'article de Caroline Durand, portant sur le discours journalistique face à l'engagement politique de plusieurs 
chanteurs et chanteuses québécois des années 1960-1970, et l'étude de Cécile Prévost-Thomas, qui a pour dessein de reproblématiser "la dénégation d'une réalité sociale" (p. 139) dans la chanson française engagée telle qu'elle se manifeste à nouveau ces dernières années dans un contexte identitaire propre à la France. Examinant la réception par la presse de figures emblématiques comme Pauline Julien, Gilles Vigneault et Raymond Lévesque, entre autres, considérant également les répercussions des fêtes nationales (la Saint-Jean-Baptiste, la Fête du Canada) et des grands rassemblements collectifs, Caroline Durand constate que «le contexte politique de l'époque influence surtout le discours de la presse artistique sur la politisation et l'engagement des artisans de la chanson " (p. 130) et que l'image véhiculée par le discours journalistique est celle d'une "société politisée" (p. 132). C'est contre le discours globalisant des médias français ignorant le plus souvent le caractère diversifié de la chanson française contemporaine, niant plus précisément l'existence d'une chanson contestataire, que s'indigne Cécile Prévost-Thomas par l'interrogation des enjeux identitaires et sociaux de la chanson engagée. Prenant pour appui les chansons "Douce France", dans la reprise par le groupe Carte de séjour, et "Tekitoi", chanson titre de l'album éponyme de Rachid Taha, la sociologue pose que "la question identitaire" telle que soulevée dans la chanson française et francophone est une "problématique sociologique" (p. 152) qu'il s'agit d'envisager de façon large pour comprendre les idées, les visions et les revendications véhiculées par le biais d'une "nouvelle» chanson engagée.

Ce collectif qui vient enrichir la collection "chanson/musique" des Éditions Triptyque a le défaut de ses qualités. Pour la plupart, les contributions ici réunies reprennent, modifient et réorientent les idées maîtresses développées dans le contexte d'un mémoire de maîtrise ou d'une thèse de doctorat. Aussi la théorie prévaut-elle parfois sur la défense et l'illustration d'une problématique précise, sur l'analyse cantologique $\mathrm{e}^{12} \mathrm{du}$ corpus à l'étude. Ou à l'inverse, il peut arriver que les idées ne trouvent pas véritablement une assise solide dans des concepts et notions théoriques.

$$
+4
$$

12. Je reprends à mon compte ce terme mis au point par Stéphane HIRSCHI dans les nombreuses publications consacrées à la chanson française et francophone (Jacques Brel. Chant contre silence, Paris, Libraire A.-G. Nizet, 1995; La chanson en lumiere, Colloque international des 24-27 avril 1996 tenu à l'Université de Valenciennes, Valenciennes, Camelia, 1997; Les frontières improbables de la chanson, Valenciennes, Camelia, 2001). Nous entendons par "cancologie" l'étude de la chanson basée sur le principe eschétique que couce analyse appropriée de l'objec "chanson* doit tenir compte des diverses composantes consticutives du genre, soit les paroles, la musique, l'interprétation (par la figure du chanceur ou de la chanteuse) et l'image. 
Quant à la toilette du texte, elle est généralement soignée, bien que la mise en page ne soit pas uniformisée, notamment en ce qui concerne la structuration des articles sur le plan des intertitres (voir les contributions d'Audrée Descheneaux et de Caroline Durand). Dernier point par lequel je soulève un problème général et qui n'est donc pas une critique visant $\mathrm{La}$ chanson francophone engagke: comme pour toute publication sur la chanson, le lecteur intéressé souhaiterait avoir à sa disposition les enregistrements des chansons à l'étude ${ }^{13}$ ou d'autres éléments facilitant la compréhension du propos, comme par exemple les paroles d'une chanson dans l'annexe de l'article (voir celui de Luc Bellemare), des extraits de partition ou des schémas musicaux (tels que proposés dans l'article d'Audrée Descheneaux).

Dans l'ensemble, les réflexions fort intéressantes de ces jeunes chercheurs sur "la chanson francophone engagée " témoignent non seulement de la diversité de ce type de chanson mais également de la pluralité des approches et des méthodes d'analyse permettant de comprendre la "chanson" comme un fait social et de la saisir en même temps comme un objet d'étude sérieux. C'est une publication engagée qui répond en quelque sorte à la problématique que les auteurs se proposent d'explorer par divers moyens.

Andrea Oberhuber Université de Montréal

\section{Yvon Roberge}

Histoire des TCA au Québec.

Connaître le passé pour façonner l'avenir

Fides, Montréal, 2008.

Yvon Roberge est présenté en quatrième de couverture comme un "observateur privilégié" de l'activité et des mœurs du syndicat des Travailleurs canadiens de l'automobile (autrefois les Travailleurs unis de l'automobile), ayant "milité activement» aux TCA durant une trentaine d'années. Il veut manifestement avec ce livre offrir une histoire éducative de son

$+4$

13. Je sais bien évidemment que l'ajout d'un disque compact pose le problème des droits d'auteur et qu'il est pratiquement inimaginable de pouvoir financer ces droits qui protègent la propriété intellectuelle des créateurs. Il s'agit simplement de formuler une fois de plus ce desideratum. 